\title{
Transformation in cricket: the black African experience
}

\author{
MA Dove, ${ }^{1} \mathrm{BSc}$ (Med) Hons; CE Draper, ${ }^{1} \mathrm{PhD}$; MS Taliep, ${ }^{2} \mathrm{PhD}$; \\ J Gray, ${ }^{1,3} \mathrm{PhD}$ \\ ${ }^{1}$ Division of Exercise Science and Sports Medicine, Department of Human \\ Biology, University of Cape Town, South Africa \\ ${ }^{2}$ Department of Sports Management, Cape Peninsular University of \\ Technology, South Africa \\ ${ }^{3}$ Cricket South Africa, Wanderers Club, Johannesburg, South Africa
}

Corresponding author: MA Dove (adove@mweb.co.za)

Background: The small number of black African (BA) cricket players progressing through the talent development pathways to the elite level has been a constant concern for Cricket South Africa (CSA). Previous attempts to accelerate the development of BA players have not produced the desired results. A description of the barriers to development is imperative for appropriate interventions.

Objectives: To understand the career progression of BA cricketers in South Africa by investigating their and other key informants' perceptions, and identifying factors that may influence progress to the elite level.

Methods: A qualitative study consisting of 23 semi-structured interviews with BA players who had succeeded at the senior level $(n=11)$, and key informants $(n=12)$, was conducted. Content analysis of the data identified 92 themes, which were then aggregated into 12 categories.

Results: Five enablers (exposure to the game, education, facilities and equipment, coaching and support networks), and five barriers (team environment, quality opportunities to compete, socio-economic factors, cricket player development pathways and leadership) were identified. Two further factors (intrapersonal attributes and targets) were identified, but the differing opinions of the interviewees on the influence of these variables made it difficult to classify them. Whilst all categories were perceived to contribute in the progress of BA players to the elite level, education, support networks, quality opportunities to compete, team environment and socio-economic factors were believed to be the most important.

Conclusion: The sustainable transformation of South African cricket appears to be not only about ensuring demographically representative teams, but also about addressing barriers affecting progress that may be experienced by all players. This study highlighted the key factors that are perceived to be specific to BA cricketers.

Keywords: talent pathways, ethnicity, career progression

S Afr J Sports Med 2016;28(1):17-22. DOI:10.17159/2078-516X/2016/v28i1a479

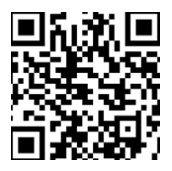

Since the onset of democracy in South Africa in 1994, the process of transforming South African sports teams to be more demographically representative has been an ongoing challenge and debate. The Transformation Status Report of 2013 found that transformation initiatives over the past 20 years had been mostly ineffectual and over-simplistic with quick fix strategies to achieve demographic change at the highest representative levels, which ignored the need for a multidimensional approach. Furthermore, it concluded that the Africanisation, (i.e. the inclusion of indigenous black Africans as opposed to people of mixed race or Indian descent) of sport has become an important focus of transformation in South African sport today. ${ }^{[1]}$

Since South Africa's return to international cricket in 1991 and the unification of the cricket bodies, firstly, as the United Cricket Board of South Africa and currently, as Cricket South Africa (CSA), there have been a number of transformation and development initiatives, including a variety of quotas and targets being set. Yet despite these interventions and a strong cricketing culture over the past 150 years, particularly in the Eastern Cape, ${ }^{[2]}$ only a limited number of black Africans (BA) have represented South Africa, and this has been largely limited to fast bowlers. ${ }^{[3]}$

The lack of representation of BA players at the provincial level and above is a cause for concern. Recent research has shown that there was a relative number of BA players transitioning from the U19 to the semi-professional and franchise categories. The number of BA players at national level then decreases disproportionally when players from the other ethnic groups are compared to the number of players at the high school level. ${ }^{[4,5]}$ Table 1 provides an overview of the racial representation of players from 2000 up to and including the $2012 / 13$ season. ${ }^{[4,5]}$

Transformation is not only about having representative numbers at all levels, but also about addressing the factors influencing the identification and development of talent. Factors contributing to elite sports performance is a growing area of research as individuals and nations strive to be the best. Previous research has shown that talent identification and development, which lead to elite sports performance are determined by an athlete's individual characteristics, influenced by genetics and training ${ }^{[6]}$ and interaction with the macroand micro-environment. ${ }^{[7]}$ CSA has recognised the need to develop more players from the BA majority ethnic group to play at the highest level. Identification of relevant barriers to BA progress in the game would assist CSA in being able to address them going forward.

The aim of this study was therefore to understand the career progression of BA cricketers in South Africa, with the specific objectives of (1) investigating the perceptions of BA players and other key informants, and (2) identifying the factors influencing both the progress to, and low representation of this group of players at the elite level.

\section{Methods}

\section{Participants and procedures}

A qualitative study was undertaken by conducting 23 semi-structured interviews. For the recruitment of participants, 22 BA male players were identified as having succeeded at the senior level. Success was defined as a player who had played at least one match of any game format at the franchise (professional) level or above, or had been awarded a franchise contract. Based on franchise representation, cricket discipline and availability, 15 players were approached to participate in the study. Eleven players (average age of 25.3 years) made themselves available to be interviewed. Four players declined the invitation to be interviewed. All players were playing at the time of the interview 
and all cricket disciplines were represented. The other 12 interviews were made up of past BA players (minimum of provincial level) who were either administrators or coaches $(\mathrm{n}=4)$, or administrators from diverse ethnic groups $(n=8)$. These participants, termed key informants, were purposively selected based on their extensive experience in the development of BA cricketers in South Africa. All six cricket franchises in South Africa were represented.

The number of interviews was believed to be sufficient, as no new trends in information were forthcoming from participants towards the end of the interview process. Each interview was conducted by the primary researcher in English, audio-recorded and lasted between 60 - and 90 minutes. The player interviews consisted of 40 interview guide questions covering topics such as their demographic profile, early cricketing experiences, career progression, personal attributes and skills, and their views on the representation of BA cricketers in South Africa. The 28 guide questions for the key informants' interviews covered their views on the career progression and the representation of BA players in South Africa.

\section{Ethical approval}

Ethical approval for the study was obtained from the University of Cape Town's Faculty of Health Sciences' Human Research Ethics Committee (HREC REF: 322/2012). All the participants provided informed consent and participated in the study voluntarily. Participant identities have not been distinguished between players and key informants to preserve anonymity, particularly of high-profile individuals.

\section{Data analysis}

The interviews were independently transcribed and the texts analysed using a content analytic approach, ${ }^{[8]}$ with the assistance of Atlas.ti Qualitative Data Analysis Software (Scientific Software Development GmbH, Berlin and Germany). During the analysis, codes were defined and derived from the data to identify themes that emerged. The number of interviewees who commented on a particular theme provided insight into the importance of that theme, and the nature of the comments provided the depth to the qualitative research. Quotations, rather than percentages, are presented to support the findings.

Table 1. Progress of players from U15 to the Proteas (national) level ${ }^{[4,5]}$

\begin{tabular}{lllllll}
\hline & $\begin{array}{l}\text { \% U15 } \\
(\mathbf{2 0 0 4 - 2 0 1 0})^{[1]}\end{array}$ & $\begin{array}{l}\text { \% U19 } \\
(2001-2009)\end{array}$ & $\begin{array}{l}\text { \% SA U19 } \\
(2000-2012)\end{array}$ & $\begin{array}{l}\text { \% Semi-Pro } \\
(\mathbf{2 0 1 2} / \mathbf{1 3})\end{array}$ & $\begin{array}{l}\text { \% Franchise } \\
(\mathbf{2 0 1 2} / \mathbf{1 3})\end{array}$ & $\begin{array}{l}\text { \% Proteas } \\
(\mathbf{2 0 1 2} / \mathbf{1 3})\end{array}$ \\
\hline $\mathrm{W}$ & 51 & 53 & 54 & 46 & 54 & 57 \\
$\mathrm{C} / \mathrm{I}$ & 23 & 24 & 28 & 33 & 32 & 37 \\
$\mathrm{BA}$ & 26 & 23 & 18 & 21 & 14 & 7 \\
\hline
\end{tabular}

Legend:

W = 'White', i.e. Caucasian or European descent; $\mathrm{C}=$ 'Coloured, i.e. mixed race or mixed ancestry;

$\mathrm{I}=$ 'Indian', i.e. Indian descent but South African in nationality; BA = 'black African', i.e. indigenous African descent

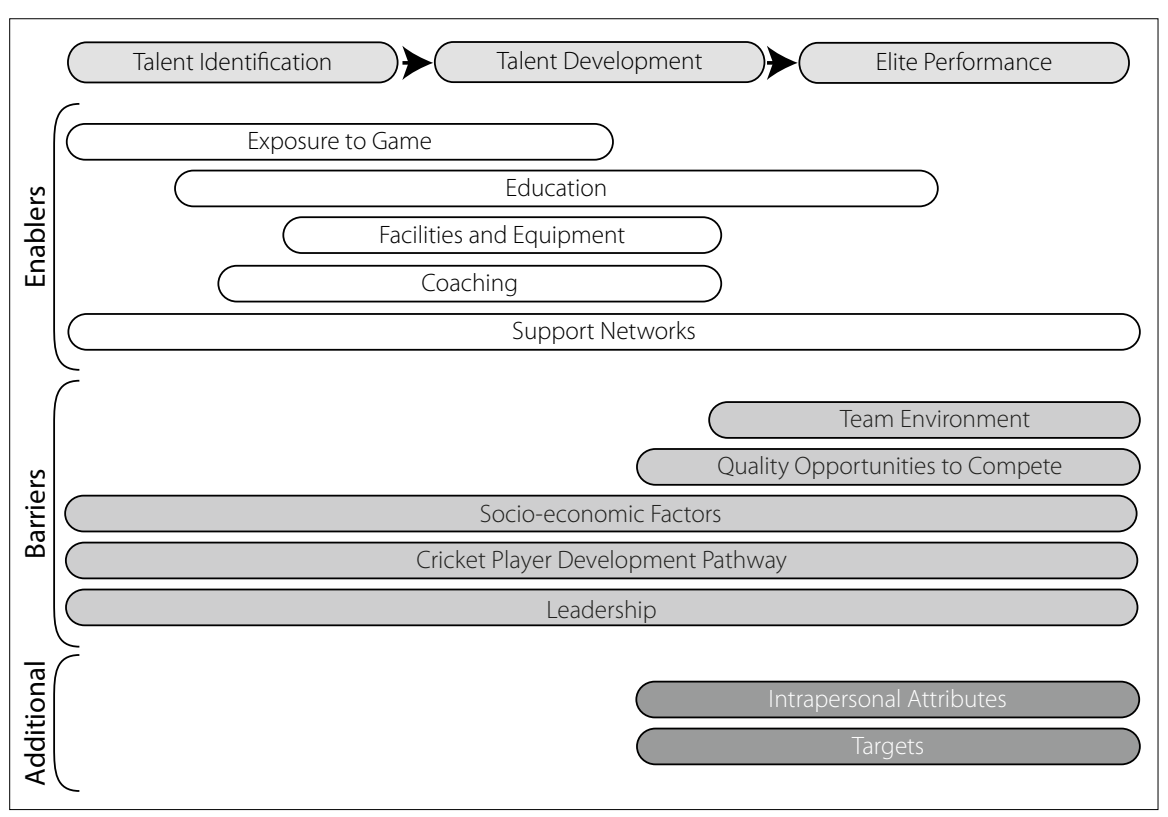

Fig. 1. Enablers and barriers along the talent pathway

Combining the players and key informants interview responses, 92 themes were identified as impacting on the ability of BA players to progress through the talent pathways. These factors could be aggregated into 12 categories, five of which were perceived as enablers to progression and five as barriers. Two further categories were difficult to classify because interviewees differed in their opinions of the influence of these variables (Figure 1). Participants acknowledged that the extent of the themes may differ between the provinces.

\section{Results and discussion}

All participants felt that there were too few BA players at the professional level and that BA batsmen were particularly poorly represented. In addition, most participants expressed concern that there was a gap in performance, and a high dropout rate among BA players between the U19 and amateur levels.

Many drop outs. There have been many that have fallen; many soldiers have fallen out the bus on this long road of ours. (Participant 6)
I think like I said at school levels you will see there is a higher number of black African players involved, but once you get out of school then they all of a sudden disappear. Maybe they're not being followed, maybe they're not being looked after as well as they should be after school, than they are at school and something has to be done to try to bridge that gap, but yes, to answer your question, there is a gap. (Participant 3)

\section{Enablers}

The participants identified the following influences that enabled them to progress to the professional level but commented that for the majority of BA players these factors were barriers to progress.

\section{Exposure to the game}

The vast majority of players interviewed grew up in a township or rural area and had early exposure (6-11 years old) to the game through a relative and/or a CSA talent scout. Thereafter their cricket skills were developed through a structured development 
programme (sponsored by Bakers Biscuits) and/or at their primary schools that had varying degrees of cricket culture. While most players also participated in other sports, the majority played provincial cricket from the U13 level upwards.

Many of the interviewees believed that seeing the game played on television was an important factor in exposing young players to cricket as it enabled them to identify role models and learn about the game. Role models provided motivation, inspiration and the belief that BA cricketers can play the game. Thus the need for more BA role models is essential, particularly BA batsmen.

The participants felt that with reduced cricket coverage on the national broadcaster's services and no formal development programme for about 10 years between the Bakers Mini Cricket programme and the current KFC-sponsored programme, there has been a decline in interest and growth of the game in BA communities leading to a reduction in numbers entering the game.

\begin{abstract}
And then what happened at school they wanted players to play Bakers Mini Cricket and I said, yes why not, let me try because I know a little bit of cricket I experienced it watching it with my uncle. And then I tried it, I played it and from there I never looked back. (Participant 16)

[...] they're all you know looking and seeing how the game's played and I think that that would be very detrimental to the black African cricketer in the townships not seeing it on TV [...]. (Participant 9)

We do not have any role model batters, there are no role models so that is one of the things that I think is a weakness. (Participant 17)
\end{abstract}

\section{Education}

Most of these players $(n=9)$ and ex-players $(n=2)$ had bursaries to attend a 'cricket-oriented' school (from 10-years-old upwards), having been identified during their younger years. They felt this was critical to their later cricket success. These schools were located outside of their immediate communities and in the former white areas, as demarcated during the apartheid era. The participants identified that the cricket culture of the school, facilities and equipment, coaching from experienced and knowledgeable coaches, a good general education, the development of life skills, and discipline were all key benefits afforded to them by this opportunity. They also mentioned that this enabled them to develop their intellectual, emotional, social and cricket intelligences on and off the field.

Despite initial challenges, such as language, cultural differences and home sickness, all the players concurred on the overall benefits of such programmes, and felt that the CSA bursary programme should be re-invigorated by providing opportunities for talented players to attend suitable 'cricket' high schools. They emphasised that the monitoring of the bursary holders' holistic progress needs to be carefully managed, as there are a number of individuals who encountered negative consequences in the past.

Participants highlighted the current poor quality of education in the majority of township and rural schools as potential limitations to progress as a cricketer in this environment. Of particular concern were disinterested teachers, lack of coaches, poor or no facilities, few quality players with whom to compete and a limited cricket culture. While the bursary system would take players out of these areas, the players felt that cricket in these areas should be developed simultaneously.

We had better education as well which was actually pretty good. I think it played a major contribution to my career. We got to learn about how to live a good life, a disciplined life, because we were at the hostel and how to make up your bed, to wear neat clothes when going to school, so it was a positive in terms of we did not only learn about cricket, but we learned about life as well which was important. (Participant 22)

\section{Facilities and equipment}

For the players interviewed, facilities and equipment were generally not considered a barrier to their cricket progress as they had access to them through the development programmes and the schools that they attended. However, it was widely acknowledged by most participants that a lack of facilities in BA communities is a barrier to the vast majority of players looking to play the sport and developing talent. While some interviewees acknowledged that facilities have been built by CSA, maintenance of these facilities has been a challenge and many are now reported to be non-functional.

The expense of batting equipment and lack of appropriate facilities were felt to have contributed to the small number of BA batsmen. Poor or no equipment was cited as having a negative psychological effect on player performance.

I think the facilities and equipment play a big role in a young, especially in a young person. I think it gives them that sort of drive to say, 'I'm playing good cricket. (Participant 18)

I remember going to U15 trip, game and I had like 2 strings of padding on mine, luckily I didn't bat, but it's so important because it just makes players comfortable. (Participant 7)

\section{Coaching}

Participants' experience with coaches differed as they transitioned through the different levels of cricket, but all players identified the positive contribution of coaches who displayed empathy, patience, passion and support. The period between U13 and U19 was considered to be the most important period for input from experienced and knowledgeable coaches. Many of the players believed that coaching was one of the most important aspects of their progress and that it enabled them to develop their talent and progress to the level they had achieved thus far. After U19, specialist coaches to develop specific aspects of a player's game were considered important.

Most participants agreed that for many BA players a lack of quality, committed coaches with adequate training and remuneration, particularly in the BA communities, were significant limitations to a player's progression through the system. This is particularly true for batsmen, who require specific technical coaching.

I think we were also fortunate enough that the coaches that we had were really committed to the program, really wanted to make a difference in the kids. (Participant 8)

[...] there is not much coaching happening in township schools in any case. (Participant 1)

\section{Support networks}

The majority of participants identified the importance of emotional support from a parent and/or significant individual from the cricket environment who assisted them to progress during their school cricket phase. However, the players indicated that this is not always true for many young BA players and together with significant financial, logistical and social pressures this compounds their difficulty in progressing in cricket.

Mentorship was seen as critical to successful progression in cricket to provide guidance, direction, nurturing and an understanding of the requirements of a top-level cricketer. Along with individual 
mentoring, ongoing life skills training and a focus on developing the 'whole' person, could potentially bridge the gap that BA players experience from the lack of a good education system. A number of respondents felt that suitably trained, experienced, empathetic and committed retired BA players would be best equipped to provide this support.

My mother and father have always been there to give advice, not necessarily on field issues, but hard to deal with things off the field. (Participant 21)

So I think, again, that mentoring program becomes critical. (Participant 8)

Previous research supports the findings of this study of the importance of a variety of support networks in the development of elite sportspersons ${ }^{[9]}$ and facilitating the transitional challenges faced at different stages of their athletic careers. ${ }^{[10]}$ The transition period between junior and senior level is particularly difficult for many sporting communities around the world. ${ }^{[10]}$ The lack of adequate support networks compounds this problem for BA players in the South African context and appropriate programmes provided by the cricketing community could assist in further developing these players.

\section{Barriers}

All the current players $(n=11)$ and the former players $(n=4)$ made their senior debut at either amateur/provincial or franchise level between the ages of 17- and 21 years old. However, only a few made significant progress and consistent selection for franchise and/or national teams. They identified the following barriers as limiting their progress.

\section{Team environment}

Most of the participants said that it was difficult for BA players to feel comfortable in the current team environments, particularly at senior level. They felt this was due to South Africa's history, differing cultures and languages, outdated mindsets, feeling misunderstood, stereotyping, poor communications, biased selections and a lack of belief and trust in BA players to perform and win games. This group of players felt that this was one of the major reasons that, having progressed to the professional level, they were unable to consistently play and deliver performances necessary to compete for places at a higher level.

The vast majority of interviewees believed that there is a need to adapt mindsets and attitudes to assist in the assimilation of different cultures into the senior teams, and to provide an environment in which all players can have a sense of belonging and support to enable them to perform to their potential.

So you buy into the talk of the team without actually owning that kind of feeling or really be feeling it. (Participant 13)

[...] You don't feel welcome and you don't feel comfortable. (Participant 15)

For example, team cohesion and culture have been linked to the sporting success of both the individual and team in professional rugby. ${ }^{[11]}$ Taylor and Bruner ${ }^{[12]}$ found a negative relationship between psychological need satisfaction and social exclusion in elite youth soccer players of black- and white-English ethnic origin. A team environment in which all players can feel comfortable to express themselves both on and off the field, and believing that they have the support of the team leadership, can only enhance both individual and team performance.

\section{Quality opportunities to compete}

Study participants felt there was a lack of quality opportunities to compete and insufficient playing time at senior level, which had an adverse effect on performance. BA players were often selected, but did not bat in the correct position or bowl sufficient overs. There was also the perception that they needed to outperform their white counterparts to retain their place in the team. Many of the players felt that they were currently experiencing a lack of quality opportunity to compete. This perception was supported by the quantitative findings of Van Zyl, ${ }^{[5]}$ and CSA have taken steps to ensure that coaches at provincial and franchise level select more BA players and play them in their appropriate roles.

A number of respondents commented that there is a perception that it is not possible to win matches with BA players in the team. At franchise and national level, winning is the priority and therefore it was felt that this is why BA players, particularly batsmen, were not selected or played in appropriate positions.

\section{They were really good, but they just didn't get the opportunity and they gave up. (Participant 6) \\ Because of the kind of inbuilt prejudice and mentalities we've spoken about people just simply always find excuses not to pick players and it still happens in franchise cricket today. (Participant 13) \\ Made 50 on debut and then sort of told to go back and work hard [...]. (Participant 16) \\ People have looked at them and said, OK, they can only bowl, they can't bat because batting tends to be associated with thinking, which I think to a larger degree, it's a deeper systemic thing that OK, black people, black cricketers wouldn't be able to think on their feet. (Participant 6)}

\section{Socio-economic factors}

All participants identified socio-economic pressures as a significant barrier to progression in cricket for the majority of BA players. While they were personally less affected by these issues, they identified gangsterism, alcohol and drug abuse, HIV/AIDS, poor nutrition, the dysfunctional education system, single- or no-parent homes and differing family priorities as barriers to success for many BA players. They indicated that lack of finances meant that many players became responsible for family members after leaving school. Accessibility to training grounds was limited by transport constraints, distances, time and money.

Some participants also mentioned that changing social recreation patterns, such as social media, amongst the youth and competition from other sports, particularly soccer and, to a lesser extent, rugby, also pose a threat to growing the game in BA areas.

They don't have the luxury of going even, getting a good education for the first 3 or 4 years because they haven't got that luxury, so they have to literally go back and go back home and get a minimum paying job and look after their families. It's a difficult one but it obviously has a lot to do with social and poverty. (Participant 19)

Do you understand this guy has got to get on two trains and three taxis and then he has to walk the last three kilometres to get here? (Participant 4)

Socio-economic factors have been acknowledged as playing a significant role in cricket participation around the world and an Australian study found that financial factors may be a barrier to participation in junior sport. ${ }^{[13]}$ While this should not detract from wanting to develop the game in all communities around South Africa, it does add an extra layer to the complex issue of socioeconomic barriers faced by BA cricketers. Many of the socio- 
economic factors mentioned are beyond the sphere of influence of the cricket administration, yet there are a number of initiatives that have and still could be implemented to increase and support cricket in BA communities. The current creation of 'hubs' and regional performance centres in the BA communities, where talented players from different schools can form well coached composite teams, with access to facilities, equipment and support structures, to compete against one another and 'traditional cricket schools', may begin to address some of the socio-economic inequalities.

\section{Cricket player development pathway}

By having exposure to a strong development programme and school system, this cohort of BA players were able to access the CSA player development pipeline. However, this is unavailable to many BA players. Many respondents expressed concern about the perceived decrease in the number of BA players entering the game at a young age, the difficulty in transitioning from U19 to senior level and the decline in participation and standard of club cricket. These factors were perceived to limit opportunities to progress or access the CSA pipeline and thus decreased representation at the senior level.

Having reached the senior level, some interviewees mentioned that BA players were treated differently from white players in terms of lower remuneration offered during contractual negotiations and a lack of investment in BA players, as senior coaches focused only on winning and not on player development. In addition, other participants said that the structure of the game at the amateur level also created barriers for BA players to progress, preventing work and study opportunities, as well as having only six professional franchises.

[...] as soon as they are out of the school structure they just disappear and you ask yourself where are they [...]? (Participant 5)

But I just see our club system being very [...] I think it's gone backwards. (Participant 9)

Stambulova et al. ${ }^{[10]}$ stated that the transition from junior to senior level is crucial for all athletes who want to reach the elite level in sport. The data presented by Van $\mathrm{Zyl}^{[5]}$ confirmed that all ethnic groups will face challenges at this stage but that these challenges appear to be greater for BA players as highlighted by their lower conversion rate to the franchise and national levels (Table 1). Therefore strategies are needed to facilitate the specific needs of BA players during this period. CSA is introducing community-based competitions to enhance the strength of club cricket. In addition, they are partnering with tertiary institutions and creating provincial academies to address the holistic development of identified players who can develop in a well-structured and supportive environment without having to deal with the socio-economic and cultural pressures on a daily basis. The efficacy of these interventions still needs to be assessed.

I think CSA should look at building what they built in Fort Hare [...] and what they have done there is then also put an education to it. I think that is a fantastic model for other people to follow. (Participant 12)

\section{Leadership}

A number of the participants felt that there was a need to encourage committed and accountable behaviours from coaches and leaders throughout the cricketing structures to enable implementation of sustainable development strategies, agreed performance standards and mindset changes at all levels.

I strongly believe up until we have the right people in the right positions, especially powerful positions, I think this whole thing will change and will start breeding more black African cricketers, just naturally without having to stress about $X, Y$ and $Z$. Have the right structures in place, at club level, at school level, everywhere. (Participant 8)

\section{Additional factors}

\section{Intrapersonal attributes}

Participants highlighted the following personal attributes that they believed contributed to cricketing success; self-belief, work ethic, mental toughness, discipline and an inner drive. Many of these have been identified in Olympic athletes that have achieved success, ${ }^{[9]}$ Australian international cricket fast bowlers ${ }^{[14]}$ and elite Australian batsmen. ${ }^{[15]}$

[...] I sort of let other factors influence me outside of cricket that actually didn't help me to perform to what I actually could have. [...] the fact of feeling inferior, the fact of not feeling good enough that had an effect on actually my performance well does not reflect what potentially I could actually do. (Participant 22)

With blacks I don't want to lie to you, these guys have got talent. Black cricketers you know they are one of the best people that I have ever come across in terms of skills, but the only problem is its up here, it is how the guys think. I think that is where the gap is in terms of here. (Participant 5)

\section{Targets}

All study participants commented on targets. Some believed they had a positive effect and others felt they had a negative effect on players progressing to the professional level. There was no consensus as to whether targets should be in place at the U19 to franchise level. The majority of respondents felt that targets should be in place at the junior levels but not at national level. It was felt by the interviewees that targets may provide opportunities to BA players that may otherwise not be given them, but there was concern for the psychological effects as a result of labelling and lack of quality from being fast-tracked.

Quotas is enabling administrators to avoid doing proper development. It's a number game. (Participant 10)

I think those targets are put there to try and help coaches make decisions which they normally don't, so sometimes it is a good thing to have those things there. How it affects the cricketer knowing that he is now a person when they talk like that, that is another debate. (Participant 17)

Quotas and targets have been a part of the South African sports transformation discussion for many years and South African cricket introduced a target of four black (BA, C and I) players at franchise and national level in 1998. This was removed in $2007 .{ }^{[3]}$ This target has resulted in increased Coloured (C) representation at franchise and national level but not BA (Table 1). It is important that interventions do not simply address demographic representation. While targets for BA cricketers increase the opportunities, they fail to address issues such as team environment and the quality of opportunity to compete, which could adversely affect performance.

\section{Limitations and further research}

One of the limitations of this study was that BA players who had not made it to the senior level were not interviewed due to difficulties in logistically identifying and contacting them. The successful players interviewed provided their perception of challenges faced by all BA players, but there may have been other barriers faced by the unsuccessful players. Furthermore, the definition used for success may have been too broad, but did allow for a consistent, less subjective 
classification of success. The players ranged from having played one match at franchise level to having represented the national team. Batting and bowling statistics may have provided more objective and narrow measures of success.

Further research is required to determine which of the themes identified are generic to all cricketers in South Africa and which are unique to BA players. The implementation of CSA initiatives aimed at developing more BA players to perform should be monitored to determine their success. The role of intrapersonal attributes in cricketing success should also be investigated.

\section{Conclusion}

This study highlighted a number of barriers and enablers to success in cricket as recalled by BA cricketers. While a number of these barriers may be generic across all ethnic groups in South Africa or even internationally, there are a number of factors which may be specific to the BA context. A recent study found similar structural barriers (socio-economic and cultural, overt racism, physical and cultural stereotypes and oppositions and organisational provision and institutional discrimination), to increased representation of ethnic minorities in European football. ${ }^{[16]}$

While it is acknowledged that increasing the BA representation at senior levels is a complex and multi-factorial issue, which includes macro- and micro- ecological factors; some of the factors identified in this study could provide insight to addressing some of the challenges identified by this study cohort. Increased exposure to the game will create opportunities to identify players for future development within an optimal environment; consisting of good education, equipment, facilities and coaching; by implementing a comprehensive bursary programme in the short term and simultaneously creating opportunities in the BA communities for long term sustainability. Enhanced support networks at all levels may better prepare BA players to compete at the elite level. At the senior level, addressing the social climate of teams, and improving the quality of opportunities to compete may enhance the performance of BA players and lower dropout rates providing a bigger pool for selection at franchise and national level.

The successful and holistic transformation of South African cricket to ensure long-term sustainability at all levels of the game will require committed leadership by all stakeholders. This will require implementation of integrated changes and adaptation of mindsets to accommodate a diversity of cultures; and in so doing provide developmental opportunities for all talented players to achieve their potential at the professional level.

Acknowledgements: The authors thank the participants for their time and cooperation in conducting this research and CSA for the funding.

\section{References}

1. Sport and Recreation South Africa (SRSA). Pilot Evaluation. Rugby, Cricket, Athletics, Netball, Football. A Transformation Status Report. 2013. [http://www.srsa. gov.za/MediaLib/Home/DocumentLibrary/SRSA] Accessed August 4, 2014.

2. Odendaal A. The story of an African game. New Africa Books. 2003.

3. CSA introduce quota for black African players. [http://www.espncricinfo.com/ southafrica/content/story/679057.html] Accessed October 14, 2013.

4. Taliep MS, Gamieldien R, West SJ. An analysis of the performance of Black African junior provincial cricket batsmen South African Journal of Sports Medicine 2011;23(3):90-94.

5. Van Zyl C. A strategy to increase the pool of black African cricket players within the CSA Pipeline to represent South Africa internationally. Final Project: Sports Management Course 2012/13. World Academy of Sport. Royal Halloway, University of London.

6. Tucker R, Collins M. What makes champions? A review of the relative contribution of genes and training to sporting success. Br J Sports Med 2012;46(8):555-561. [http://dx.doi.org/10.1136/bjsports-2011-090548]

7. Henriksen K. The ecology of talent development in sport. PhD Dissertation. University of Southern Denmark. 2010.

8. Hsieh H-F, Shannon SE. Three approaches to qualitative content analysis. Qualitative health research. Qual Health Res 2005;15(9):1277-1288. [http://dx.doi. org/10.1177/1049732305276687]

9. Gould D, Dieffenbach K, Moffett A. The development of psychological talent in U.S. Olympic champions. Final Grant Report. December 2001.

10. Stambulova N, Alfermann D, Stauer T, et al. ISSP Position Stand: Career development and transitions of athletes. IJSEP 2009;7(4):395-412. [http://dx.doi.org/10.1080/1612 197X.2009.9671916]

11. Hodge K, Henry G, Smith W. A case study of excellence in elite sport: Motivational climate in a world champion team. The Sport Psychologist 2014;28:60-74. [http:// dx.doi.org/10.1123/tsp.2013-0037]

12. Taylor IM, Bruner MW. The social environment and developmental experiences in elite youth soccer. Psychology of Sport and Exercise 2012;13(4):390-396. [http:// dx.doi.org/10.1016/j.psychsport.2012.01.008]

13. Kirk D, Carlson T, O'Connor A, et al. The economic impact on families of children's participation in junior sport. Aust J Sci Med Sport 1997;29(2):27-33.

14. Phillips E, Davids K, Renshaw I, et al. Acquisition of expertise in cricket fast bowling: perceptions of expert players and coaches. J Sci Med Sport 2013;17(1):85-90. [http:// dx.doi.org/10.1016/j.jsams.2013.03.005]

15. Weissensteiner JR, Abernethy B, Farrow D, et al. Distinguishing psychological characteristics of expert cricket batsmen. J Sci Med Sport 2012;15(1):74-79. [http:// dx.doi.org/10.1016/j.jsams.2011.07.003]

16. Bradbury S, Amara M, Garcia B, et al. Representation and structural discrimination in football in Europe. The case of minorities and women. Loughborough: Loughborough University, 2011. 